\title{
Assessment of age at menarche and menstrual pattern among secondary school girls in Awka, Southeast Nigeria
}

\section{Cyril C. Ezenyeaku*, Ifeanyichukwu U. Ezebialu, Joseph C. Umeobika, Josephat Akabike, Chijioke A. Ezenyeaku, Chukwuemeka E. Ojiyi, Tochukwu C. Okeke}

\begin{abstract}
Department of Obstetrics and Gynaecology, Chukwuemeka Odumegwu Ojukwu University Teaching Hospital, Awka,
\end{abstract} Nigeria

Received: 09 April 2021

Accepted: 05 May 2021

*Correspondence:

Dr. Cyril C. Ezenyeaku,

E-mail: cyrilezenyeaku@yahoo.com

Copyright: (c) the author(s), publisher and licensee Medip Academy. This is an open-access article distributed under the terms of the Creative Commons Attribution Non-Commercial License, which permits unrestricted non-commercial use, distribution, and reproduction in any medium, provided the original work is properly cited.

\begin{abstract}
Background: Menarche is an important physiological milestone in girls and this usually occurs during their secondary school years. The menstrual pattern may vary in these adolescents. This knowledge may assist in counselling the young girls on reproductive health issues.

Methods: A cross-sectional study of female secondary school students from Kenneth Dike secondary school, Awka; Amaenyi girls' secondary school and community secondary school, Okpuno, all in Awka, Anambra state of Nigeria. All together 545 students were selected following informed consent. The study instrument was a pre-tested, semistructured and self-administered questionnaire. Data analysis was done with IBM SPSS 20 software. Tests of association between variables were done with Fisher's exact test and the level of significance set at $\mathrm{p} \leq 0.05$.

Results: The mean age of the 545 respondents was $16.79 \pm 1.736$ years and majority of them are of the Igbo ethnic group $(90.5 \%)$. The average age at menarche was $12.77 \pm 1.159$ years. There is a significant difference between the age at menarche and the father's occupation. The modal duration of flow was 4 days $(48.7 \%)$ while $95.2 \%$ had a normal cycle length of 21-35 days.

Conclusions: The average age of menarche of secondary school girls in Awka was 12.77 \pm 1.159 years. Most of the students had normal menstrual flow duration of 2-7 days with a cycle length of 21-35 days.
\end{abstract}

Keywords: Age, Menarche, Menstrual pattern, School, Awka

\section{INTRODUCTION}

Menarche is an important milestone in the reproductive life of a woman. It is the onset of the very first menstruation of a young woman. ${ }^{1}$ Menstruation is a normal physiological process that begins during adolescence and may be associated with various features occurring before or during the menstrual flow. ${ }^{1}$ It is the culmination of a cascade of physiological events involving the pituitary gland, the ovaries and the uterus. Age at menarche is known to be influenced by many factors including genetics, nutrition, sporting activities, environment and economic status and may have geographical variation. ${ }^{2-4}$ Various studies have reported varying ages at menarche in different populations. Ikaraoha in Rivers state of Nigeria reported 13.19 \pm 1.32 years for urban and 14.22 years for rural areas while a Kano study among secondary school students gave a mean age at menarche of 12.8 years. ${ }^{5,6}$ There was no statistically significant association between the age at menarche and the socioeconomic status of the parents in these studies., 5 Another study at Abakaliki observed a mean age at menarche of $13 \pm 1.0$ years and with a high socioeconomic status of parents tending to reduce the age among the participants. ${ }^{7}$ Diorgu reported a mean menarcheal age of $12.8 \pm 1.6$ years in Portharcourt. ${ }^{8}$ Pandez in Mozambique reported $13.9 \pm 1.29$ years. $^{9}$ 
However, menarcheal age of 13.66 years was reported in Morocco. ${ }^{10}$

Even though, biology of the human reproductive system is taught in Nigerian secondary schools, cultural and family inhibitions have restricted open discussion of menstrual issues especially with adolescents. ${ }^{6}$ However, adolescent girls and young women still discuss menstrual issues with their mothers and other close female associates.

In a Gambian study, two thirds of the surveyed girls reported having learnt about menstruation before menarche; however at menarche most felt unprepared. ${ }^{11}$ Teachers were the main source of information, but when asking for advice most girls preferred to ask their mothers. Mothers reported facing difficulties in discussing menstruation with their children and felt that boys did not need to be taught about it. Most girls used reusable cloth unless they are given free menstrual pads from school. ${ }^{11}$

In the adolescent, with onset of menstruation, the cycle and pattern of flow may take some period of time to stabilize. Some may have abnormally long or short cycles and volume of flow may vary as well. ${ }^{12}$ A study in Northwest Nigeria showed that about one-quarter of the adolescent girls had a cycle length of 28 days. The average duration of flow was $4.9 \pm 5.1$ days with a normal amount of blood flow and regular menses in more than $80 \%$ of the adolescent school girls. ${ }^{13}$ In a similar study in Portharcourt, South Nigeria, the average duration of the menstrual cycle was $29.8 \pm 3.3$ days. $^{8}$

Though studies have been done on various aspects of menarche and menstrual life of school girls in different parts of the world and the country, none has been documented in Awka, Southeast Nigeria which is the capital city of Anambra state and the seat of a teaching hospital and a medical college, Chukwuemeka Odumegwu Ojukwu university college of medicine and its affiliated teaching hospital. This study was aimed at finding the age at menarche and the menstrual pattern of secondary school girls at Awka, Southeast Nigeria. The results and findings will be a good tool in the hands of reproductive health practitioners in counselling and management of related conditions in these adolescents.

\section{METHODS}

Current study was conducted amongst girls drawn from post-primary schools in Awka, the capital city of Anambra state of Southeast Nigeria in May 2019. Many secondary schools in Awka are characterized by the absence of boarding facilities with the resultant high numbers of day students. Three post-primary schools were selected at random Kenneth Dike secondary school, Awka; Amaenyi girls' secondary school and community secondary school, Okpuno. Kenneth Dike secondary school and community secondary school, Okpuno are co- educational institutions while the other is a female secondary school.

The survey employed a cross-sectional, descriptive study design. One class of a stream was selected at random starting from junior secondary school II (JSS II) to senior secondary school III (SSS III). The JSS I was excluded because it usually consists of very young children who are in their very first experience of post-primary education and are not yet in tune with secondary school life. Secondary school education in Nigeria is structured in a way that students spend a total of six years. The first three years are spent in the junior secondary segment while the last three are spent in the senior secondary segment. JSS II stream of a school may have up to three classes for instance, and only one class was selected. In the co-educational school, obviously only the female students in the selected classes were included in the study.

All together 545 students were drawn following informed consent. Their parents/guardians also gave their permission as well. The study instrument was a pretested, semi-structured and self-administered questionnaire which contained questions on sociodemographic characteristics including age, father's occupation and mother's level of educational attainment, religion and tribe. The father's occupation classification as a measure of socio-economic status was that developed by Oyedeji. ${ }^{14}$ Also contained in the questionnaire were height and weight (which were measured by the research assistant), age at menarche, duration of flow, presence of heavy flow, cycle length, dysmenorrhoea, etc. The weight and height were measured as the questionnaire was being administered. These were used to calculate their body mass index (BMI). Some of the selected students had not achieved menarche and therefore did not finish all the questions contained in the questionnaire. Data analysis was done with IBM SPSS 20 software. Tests of association between variables were done with Fisher's exact test and the level of significance set at $\mathrm{p} \leq 0.05$.

\section{RESULTS}

The mean age of the 545 respondents was $16.79 \pm 1.736$ years and majority of them are of the Igbo ethnic group $(90.5 \%)$. Most of them were in the 15-17 years age group. Seventy one percent of the respondents had normal body weight (BMI of $18.5-24.9 \mathrm{~kg} / \mathrm{m}^{2}$ ) while only $1.3 \%$ of them were obese. The rest of the sociodemographic characteristics of the respondents are shown in (Table 1). The age at menarche of the respondents is shown in (Table 2). The average age at menarche was $12.77 \pm 1.159$ years. The modal age was 12 years.

Most of the respondents $(83.9 \%)$ informed their mothers first about the onset of menarche even though a lesser number got pre-menarcheal education from them (67.2\%) as shown in (Table 3). 
There was a significant difference between the age at menarche and the father's occupation (Table 4). But there was no significant difference between the flow duration and the respondents' BMI, presence of dysmenorrhoea or the cycle length (Table 5).

The menstrual pattern is shown in (Table 6). The modal duration of flow was 4 days $(48.7 \%)$ while $95.2 \%$ had a normal cycle length of 21-35 days.

Table 1: Socio-demographic characteristics of the respondents.

\begin{tabular}{|c|c|c|}
\hline Variables $(n=545)$ & $\mathbf{N}$ & $(\%)$ \\
\hline \multicolumn{3}{|l|}{ Age（years) } \\
\hline $12-14$ & 43 & 7.9 \\
\hline $15-17$ & 323 & 59.3 \\
\hline $18-20$ & 168 & 30.8 \\
\hline $21-23$ & 11 & 2.0 \\
\hline Mean \pm SD & \multicolumn{2}{|c|}{$16.79 \pm 1.736$} \\
\hline Minimum; maximum & \multicolumn{2}{|c|}{$12 ; 22$} \\
\hline \multicolumn{3}{|l|}{ BMI } \\
\hline Underweight & 91 & 16.7 \\
\hline Normal weight & 387 & 71.0 \\
\hline Overweight & 60 & 11.0 \\
\hline Obese & 7 & 1.3 \\
\hline \multicolumn{3}{|l|}{ Tribe } \\
\hline Igbo & 493 & 90.5 \\
\hline Hausa & 5 & 0.9 \\
\hline Yoruba & 10 & 1.8 \\
\hline Others & 37 & 6.8 \\
\hline \multicolumn{3}{|l|}{ Religion } \\
\hline Anglican & 104 & 19.1 \\
\hline Roman Catholic & 255 & 46.8 \\
\hline Pentecostal & 183 & 33.6 \\
\hline Islam & 3 & 0.6 \\
\hline Others & 0 & 0 \\
\hline \multicolumn{3}{|l|}{ Father's occupation } \\
\hline Professional & 79 & 14.5 \\
\hline Administrative officer & 60 & 11.0 \\
\hline Artisan & 107 & 19.6 \\
\hline Business man & 229 & 42.0 \\
\hline Farmer & 70 & 12.8 \\
\hline \multicolumn{3}{|c|}{ Mother's educational status } \\
\hline No formal education & 18 & 3.3 \\
\hline Primary & 76 & 13.9 \\
\hline Secondary & 265 & 48.6 \\
\hline Tertiary & 186 & 34.1 \\
\hline \multicolumn{3}{|l|}{ Resides with whom? } \\
\hline Parents & 313 & 57.4 \\
\hline Relatives & 57 & 10.5 \\
\hline Guardian & 163 & 29.9 \\
\hline Step mother & 7 & 1.3 \\
\hline Others & 5 & 0.9 \\
\hline
\end{tabular}

Table 2: Age at menarche of respondents.

\begin{tabular}{|lll|}
\hline Variables & Frequency & $(\%)$ \\
\hline Achieved menarche? (N=545) & & \\
\hline Yes & 503 & 92.3 \\
\hline No & 42 & 7.7 \\
\hline Age at menarche (years) & $\mathbf{( N = 5 0 3 )}$ & \\
\hline 9 & 3 & 0.6 \\
\hline 10 & 11 & 2.2 \\
\hline 11 & 36 & 7.2 \\
\hline 12 & 168 & 33.4 \\
\hline 13 & 152 & 30.2 \\
\hline 14 & 100 & 19.9 \\
\hline 15 & 31 & 6.2 \\
\hline 16 & 2 & 0.4 \\
\hline
\end{tabular}

Table 3: Characteristics and events around menarche of the respondents.

\begin{tabular}{|c|c|c|}
\hline Variables & Frequency & $(\%)$ \\
\hline \multicolumn{3}{|c|}{ First person informed of menarche $(\mathrm{N}=503)$} \\
\hline Mother & 422 & 83.9 \\
\hline Teacher & 4 & 0.8 \\
\hline Girlfriend & 22 & 4.4 \\
\hline Others & 55 & 10.9 \\
\hline \multicolumn{3}{|c|}{ Educated on menses prior to menarche $(\mathrm{N}=503)$} \\
\hline Yes & 469 & 93.2 \\
\hline No & 34 & 6.8 \\
\hline \multicolumn{3}{|c|}{ Pre-menarche education given by $(\mathrm{N}=469)$} \\
\hline Mother & 315 & 67.2 \\
\hline Sister & 53 & 11.3 \\
\hline Teacher & 71 & 15.1 \\
\hline Friend & 12 & 2.6 \\
\hline Others & 18 & 3.8 \\
\hline \multicolumn{3}{|c|}{ Received post-menarche education $(\mathrm{N}=501)$} \\
\hline Yes & 457 & 91.2 \\
\hline No & 44 & 8.8 \\
\hline \multicolumn{3}{|c|}{$\begin{array}{l}\text { Ever discussed menstruation with your mother } \\
(\mathrm{N}=503)\end{array}$} \\
\hline Yes & 472 & 93.8 \\
\hline No & 31 & 6.2 \\
\hline
\end{tabular}

\section{DISCUSSION}

Current study showed an average age at menarche of $12.77 \pm 1.159$ years and is similar to the findings of $13.19 \pm 1.32$ years in a Portharcourt study and 12.8 years in other Portharcourt and Kano studies. ${ }^{5,6,8}$ It is also similar to the reports from other African countries of Mozambique and Morocco. ${ }^{9,10}$ There is also an agreement with the mean menarcheal age of 12.52 years found in an Indian study. ${ }^{15}$ The significant association between the father's occupation and the age at menarche is expected as this is a reflection of the socio-economic status which is known to have an inverse relationship with the age at menarche. 
Table 4: Association between age at menarche and socio-demographic characteristics of the respondents.

\begin{tabular}{|c|c|c|c|c|c|c|c|c|c|c|}
\hline \multirow{2}{*}{$\begin{array}{l}\text { Variables } \\
(\mathrm{n}=503)\end{array}$} & \multicolumn{8}{|c|}{ Age at menarche (years) $\mathbf{N}(\%)$} & \multirow{2}{*}{$\begin{array}{l}\text { Test } \\
\text { statistics } \\
\text { F value }\end{array}$} & \multirow{2}{*}{ P value } \\
\hline & 9 & 10 & 11 & 12 & 13 & 14 & 15 & 16 & & \\
\hline \multicolumn{9}{|l|}{ BMI } & \multirow{5}{*}{16.870} & \multirow{5}{*}{0.719} \\
\hline $\begin{array}{l}<18.5 \\
\text { (Underweight) }\end{array}$ & $\begin{array}{l}0 \\
(0.0)\end{array}$ & $\begin{array}{l}3 \\
(4.1)\end{array}$ & $\begin{array}{l}3 \\
(4.1)\end{array}$ & $\begin{array}{l}24 \\
(32.9)\end{array}$ & $\begin{array}{l}22 \\
(30.1)\end{array}$ & $\begin{array}{l}13 \\
(17.8)\end{array}$ & $\begin{array}{l}7 \\
(9.6)\end{array}$ & $\begin{array}{l}1 \\
(1.4)\end{array}$ & & \\
\hline $\begin{array}{l}18.5-24.9 \\
\text { (Normal weight) }\end{array}$ & $\begin{array}{l}2 \\
(0.5)\end{array}$ & $\begin{array}{l}6 \\
(1.6)\end{array}$ & $\begin{array}{l}28 \\
(7.7)\end{array}$ & $\begin{array}{l}124 \\
(34.1)\end{array}$ & $\begin{array}{l}111 \\
(30.5)\end{array}$ & $\begin{array}{l}76 \\
(20.9)\end{array}$ & $\begin{array}{l}16 \\
(4.4)\end{array}$ & $\begin{array}{l}1 \\
(0.3)\end{array}$ & & \\
\hline $\begin{array}{l}25.0-29.9 \\
\text { (Overweight) }\end{array}$ & $\begin{array}{l}1 \\
(1.7)\end{array}$ & $\begin{array}{l}2 \\
(3.4)\end{array}$ & $\begin{array}{l}4 \\
(6.8)\end{array}$ & $\begin{array}{l}18 \\
(30.5)\end{array}$ & $\begin{array}{l}18 \\
(30.5)\end{array}$ & $\begin{array}{l}9 \\
(15.3)\end{array}$ & $\begin{array}{l}7 \\
(11.9)\end{array}$ & $\begin{array}{l}0 \\
(0.00)\end{array}$ & & \\
\hline $\begin{array}{l}\geq 30 \\
\text { (Obese) }\end{array}$ & $\begin{array}{l}0 \\
(0.0)\end{array}$ & $\begin{array}{l}0 \\
(0.0)\end{array}$ & $\begin{array}{l}1 \\
(14.3)\end{array}$ & $\begin{array}{l}2 \\
(28.6)\end{array}$ & $\begin{array}{l}1 \\
(14.3)\end{array}$ & $\begin{array}{l}2 \\
(28.6)\end{array}$ & $\begin{array}{l}1 \\
(14.3)\end{array}$ & $\begin{array}{l}0 \\
(0.0)\end{array}$ & & \\
\hline \multicolumn{9}{|l|}{ Tribe } & \multirow{5}{*}{17.009} & \multirow{5}{*}{0.711} \\
\hline Igbo & $\begin{array}{l}3 \\
(0.7)\end{array}$ & $\begin{array}{l}10 \\
(2.2)\end{array}$ & $\begin{array}{l}34 \\
(7.4)\end{array}$ & $\begin{array}{l}157 \\
(34.4)\end{array}$ & $\begin{array}{l}133 \\
(29.1)\end{array}$ & $\begin{array}{l}90 \\
(19.7)\end{array}$ & $\begin{array}{l}28 \\
(6.1)\end{array}$ & $\begin{array}{l}2 \\
(0.4)\end{array}$ & & \\
\hline Hausa & $\begin{array}{l}0 \\
(0.0)\end{array}$ & $\begin{array}{l}0 \\
(0.0)\end{array}$ & $\begin{array}{l}0 \\
(0.0)\end{array}$ & $\begin{array}{l}0 \\
(0.0)\end{array}$ & $\begin{array}{l}2 \\
(50.0)\end{array}$ & $\begin{array}{l}2 \\
(50.0)\end{array}$ & $\begin{array}{l}0 \\
(0.0)\end{array}$ & $\begin{array}{l}0 \\
(0.0)\end{array}$ & & \\
\hline Yoruba & $\begin{array}{l}0 \\
(0.0)\end{array}$ & $\begin{array}{l}0 \\
(0.0)\end{array}$ & $\begin{array}{l}0 \\
(0.0)\end{array}$ & $\begin{array}{l}0 \\
(0.0) \\
\end{array}$ & $\begin{array}{l}7 \\
(77.8)\end{array}$ & $\begin{array}{l}2 \\
(22.2)\end{array}$ & $\begin{array}{l}0 \\
(0.0)\end{array}$ & $\begin{array}{l}0 \\
(0.0)\end{array}$ & & \\
\hline Others & $\begin{array}{l}0 \\
(0.0)\end{array}$ & $\begin{array}{l}1 \\
(3.0)\end{array}$ & $\begin{array}{l}2 \\
(6.1)\end{array}$ & $\begin{array}{l}11 \\
(33.3)\end{array}$ & $\begin{array}{l}10 \\
(30.0)\end{array}$ & $\begin{array}{l}6 \\
(18.2)\end{array}$ & $\begin{array}{l}3 \\
(9.1)\end{array}$ & $\begin{array}{l}0 \\
(0.0)\end{array}$ & & \\
\hline \multicolumn{9}{|c|}{ Mother's educational status } & \multirow{5}{*}{10.306} & \multirow{5}{*}{0.975} \\
\hline $\begin{array}{l}\text { No formal } \\
\text { education }\end{array}$ & $\begin{array}{l}0 \\
(0.0)\end{array}$ & $\begin{array}{l}0 \\
(0.0)\end{array}$ & $\begin{array}{l}1 \\
(5.6)\end{array}$ & $7(38.9)$ & $\begin{array}{l}6 \\
(33.3)\end{array}$ & $\begin{array}{l}3 \\
(16.7)\end{array}$ & $\begin{array}{l}1 \\
(5.6)\end{array}$ & $\begin{array}{l}0 \\
(0.0)\end{array}$ & & \\
\hline Primary & $\begin{array}{l}0 \\
(0.0)\end{array}$ & $\begin{array}{l}2 \\
(2.7)\end{array}$ & $\begin{array}{l}6 \\
(8.1)\end{array}$ & $\begin{array}{l}19 \\
(25.7)\end{array}$ & $\begin{array}{l}26 \\
(35.1)\end{array}$ & $\begin{array}{l}15 \\
(20.3)\end{array}$ & $\begin{array}{l}5 \\
(6.8)\end{array}$ & $\begin{array}{l}1 \\
(1.4)\end{array}$ & & \\
\hline Secondary & $\begin{array}{l}3 \\
(1.2)\end{array}$ & $\begin{array}{l}5 \\
(2.0)\end{array}$ & $\begin{array}{l}15 \\
(6.1)\end{array}$ & $\begin{array}{l}88 \\
(36.1)\end{array}$ & $\begin{array}{l}69 \\
(28.3)\end{array}$ & $\begin{array}{l}49 \\
(20.1)\end{array}$ & $\begin{array}{l}14 \\
(5.7)\end{array}$ & $\begin{array}{l}1 \\
(0.4)\end{array}$ & & \\
\hline Tertiary & $\begin{array}{l}0 \\
(0.0)\end{array}$ & $\begin{array}{l}4 \\
(2.4)\end{array}$ & $\begin{array}{l}14 \\
(8.4)\end{array}$ & $\begin{array}{l}54 \\
(32.3)\end{array}$ & $\begin{array}{l}51 \\
(30.5)\end{array}$ & $\begin{array}{l}33 \\
(19.8)\end{array}$ & $\begin{array}{l}11 \\
(6.6)\end{array}$ & $\begin{array}{l}0 \\
(0.0)\end{array}$ & & \\
\hline \multicolumn{9}{|l|}{ Resides with } & \multirow{6}{*}{26.608} & \multirow{6}{*}{0.540} \\
\hline Parents & $\begin{array}{l}2 \\
(0.7)\end{array}$ & $\begin{array}{l}6 \\
(2.1)\end{array}$ & $\begin{array}{l}22 \\
(7.6)\end{array}$ & $\begin{array}{l}103 \\
(35.4)\end{array}$ & $\begin{array}{l}84 \\
(28.9)\end{array}$ & $\begin{array}{l}55 \\
(18.9)\end{array}$ & $\begin{array}{l}19 \\
(6.5)\end{array}$ & $\begin{array}{l}0 \\
(0.0)\end{array}$ & & \\
\hline Relatives & $\begin{array}{l}0 \\
(0.0)\end{array}$ & $\begin{array}{l}3 \\
(5.8)\end{array}$ & $\begin{array}{l}2 \\
(3.8)\end{array}$ & $\begin{array}{l}21 \\
(40.4)\end{array}$ & $\begin{array}{l}15 \\
(28.8)\end{array}$ & $\begin{array}{l}8 \\
(15.4)\end{array}$ & $\begin{array}{l}2 \\
(3.8)\end{array}$ & $\begin{array}{l}1 \\
(1.9)\end{array}$ & & \\
\hline Guardian & $\begin{array}{l}1 \\
(0.7)\end{array}$ & $\begin{array}{l}2 \\
(1.3)\end{array}$ & $\begin{array}{l}12 \\
(7.9)\end{array}$ & $\begin{array}{l}43 \\
(28.5)\end{array}$ & $\begin{array}{l}49 \\
(32.5)\end{array}$ & $\begin{array}{l}34 \\
(22.5)\end{array}$ & $\begin{array}{l}9 \\
(6.0)\end{array}$ & $\begin{array}{l}1 \\
(0.7)\end{array}$ & & \\
\hline Step-mother & $\begin{array}{l}0 \\
(0.0)\end{array}$ & $\begin{array}{l}0 \\
(0.0)\end{array}$ & $\begin{array}{l}0 \\
(0.0)\end{array}$ & $\begin{array}{l}1 \\
(20.0)\end{array}$ & $\begin{array}{l}1 \\
(20.0)\end{array}$ & $\begin{array}{l}3 \\
(60.0)\end{array}$ & $\begin{array}{l}0 \\
(0.0)\end{array}$ & $\begin{array}{l}0 \\
(0.0)\end{array}$ & & \\
\hline Others & $\begin{array}{l}0 \\
(0.0)\end{array}$ & $\begin{array}{l}0 \\
(0.0)\end{array}$ & $\begin{array}{l}0 \\
(0.0)\end{array}$ & $\begin{array}{l}0 \\
(0.0)\end{array}$ & $\begin{array}{l}3 \\
(75.0)\end{array}$ & $\begin{array}{l}0 \\
(0.0)\end{array}$ & $\begin{array}{l}1 \\
(25.0)\end{array}$ & $\begin{array}{l}0 \\
(0.0)\end{array}$ & & \\
\hline \multicolumn{9}{|c|}{ Father's occupation } & \multirow{6}{*}{46.797} & \multirow{6}{*}{0.014} \\
\hline Professional & $\begin{array}{l}0 \\
(0.0)\end{array}$ & $\begin{array}{l}4 \\
(5.5)\end{array}$ & $\begin{array}{l}9 \\
(12.3)\end{array}$ & $\begin{array}{l}23 \\
(31.5)\end{array}$ & $\begin{array}{l}21 \\
(28.8)\end{array}$ & $\begin{array}{l}15 \\
(20.5)\end{array}$ & $\begin{array}{l}1 \\
(1.4)\end{array}$ & $\begin{array}{l}0 \\
(0.0)\end{array}$ & & \\
\hline $\begin{array}{l}\text { Administrative } \\
\text { officer }\end{array}$ & $\begin{array}{l}1 \\
(1.8)\end{array}$ & $\begin{array}{l}1 \\
(1.8)\end{array}$ & $\begin{array}{l}1 \\
(1.8)\end{array}$ & $\begin{array}{l}15 \\
(27.3)\end{array}$ & $\begin{array}{l}18 \\
(32.7)\end{array}$ & $\begin{array}{l}13 \\
(23.6)\end{array}$ & $\begin{array}{l}6 \\
(10.9)\end{array}$ & $\begin{array}{l}0 \\
(0.0)\end{array}$ & & \\
\hline Artisan & $\begin{array}{l}1 \\
(1.1)\end{array}$ & $\begin{array}{l}5 \\
(5.4)\end{array}$ & $\begin{array}{l}3 \\
(3.2)\end{array}$ & $\begin{array}{l}26 \\
(28.0)\end{array}$ & $\begin{array}{l}32 \\
(34.4)\end{array}$ & $\begin{array}{l}15 \\
(16.1)\end{array}$ & $\begin{array}{l}11 \\
(11.8)\end{array}$ & $\begin{array}{l}0 \\
(0.0)\end{array}$ & & \\
\hline Business man & $\begin{array}{l}1 \\
(0.5)\end{array}$ & $\begin{array}{l}1 \\
(0.5)\end{array}$ & $\begin{array}{l}16 \\
(7.5)\end{array}$ & $\begin{array}{l}83 \\
(38.8)\end{array}$ & $\begin{array}{l}63 \\
(29.4)\end{array}$ & $\begin{array}{l}43 \\
(20.1)\end{array}$ & $\begin{array}{l}6 \\
(2.8)\end{array}$ & $\begin{array}{l}1 \\
(0.5)\end{array}$ & & \\
\hline Farmer & $\begin{array}{l}0 \\
(0.0)\end{array}$ & $\begin{array}{l}0 \\
(0.0)\end{array}$ & $\begin{array}{l}7 \\
(10.3)\end{array}$ & $\begin{array}{l}21 \\
(30.9)\end{array}$ & $\begin{array}{l}18 \\
(26.5)\end{array}$ & $\begin{array}{l}14 \\
(20.6)\end{array}$ & $\begin{array}{l}7 \\
(10.3)\end{array}$ & $\begin{array}{l}1 \\
(1.5)\end{array}$ & & \\
\hline
\end{tabular}


Table 5: Association between flow duration and some selected factors.

\begin{tabular}{|c|c|c|c|c|}
\hline \multirow{2}{*}{$\begin{array}{l}\text { Variable } \\
(\mathbf{N}=\mathbf{5 0 3})\end{array}$} & \multicolumn{2}{|c|}{$\begin{array}{l}\text { Flow duration (days) } \\
\mathbf{N}(\%)\end{array}$} & \multirow{2}{*}{$\begin{array}{l}\text { Test statistics } \\
\text { F value }\end{array}$} & \multirow[t]{2}{*}{$P$ value } \\
\hline & $1-7$ & $\geq 8$ & & \\
\hline \multicolumn{3}{|l|}{ BMI } & \multirow{5}{*}{1.146} & \multirow{5}{*}{0.766} \\
\hline$<18.5$ (Underweight) & $72(98.6)$ & $1(1.4)$ & & \\
\hline $18.5-24.9$ (Normal weight ) & $362(99.5)$ & $2(0.5)$ & & \\
\hline $25.0-29.9$ (Overweight) & $59(100.0)$ & $0(0.0)$ & & \\
\hline$\geq 30$ (Obese) & $7(100.0)$ & $0(0.0)$ & & \\
\hline \multicolumn{3}{|l|}{ Tires easily (anaemia) } & \multirow{3}{*}{0.744} & \multirow{3}{*}{0.388} \\
\hline Yes & $290(99.7)$ & $1(0.3)$ & & \\
\hline No & $210(99.1)$ & $2(0.9)$ & & \\
\hline \multicolumn{3}{|l|}{ Heavy flow } & \multirow{3}{*}{0.523} & \multirow{3}{*}{0.470} \\
\hline Yes & $229(99.1)$ & $2(0.9)$ & & \\
\hline No & $271(99.6)$ & $1(0.4)$ & & \\
\hline \multicolumn{3}{|l|}{ Menstruates monthly } & \multirow{3}{*}{2.371} & \multirow{3}{*}{0.124} \\
\hline Yes & 458 (99.6) & $2(0.4)$ & & \\
\hline No & $42(97.7)$ & $1(2.3)$ & & \\
\hline \multicolumn{3}{|l|}{ Dysmenorrhoea } & \multirow{3}{*}{2.624} & \multirow{3}{*}{0.105} \\
\hline Yes & $372(99.7)$ & $1(0.3)$ & & \\
\hline No & $128(98.5)$ & $2(1.5)$ & & \\
\hline
\end{tabular}

Table 6: Menstrual pattern of the respondents.

\begin{tabular}{|c|c|c|}
\hline Variable $(n=503)$ & Frequency & $(\%)$ \\
\hline \multicolumn{3}{|c|}{ Menstrual flow duration (days) } \\
\hline 1 & 2 & 0.4 \\
\hline 2 & 3 & 0.6 \\
\hline 3 & 44 & 8.7 \\
\hline 4 & 245 & 48.7 \\
\hline 5 & 173 & 34.4 \\
\hline 6 & 17 & 3.4 \\
\hline 7 & 16 & 3.2 \\
\hline 8 & 2 & 0.4 \\
\hline 10 & 1 & 0.2 \\
\hline \multicolumn{3}{|c|}{ Tires easily after menstruation } \\
\hline Yes & 291 & 57.9 \\
\hline No & 212 & 42.1 \\
\hline \multicolumn{3}{|l|}{ Heavy flow } \\
\hline Yes & 231 & 45.9 \\
\hline No & 272 & 54.1 \\
\hline \multicolumn{3}{|c|}{ Menstrual cycle length (days) } \\
\hline$<21$ & 13 & 2.6 \\
\hline $21-35$ & 479 & 95.2 \\
\hline$>35$ & 11 & 2.2 \\
\hline \multicolumn{3}{|l|}{ Dysmenorrhoea } \\
\hline Yes & 373 & 74.2 \\
\hline No & 130 & 25.8 \\
\hline \multicolumn{3}{|c|}{ Missed school due to menses } \\
\hline Yes & 122 & 24.3 \\
\hline No & 381 & 75.7 \\
\hline \multicolumn{3}{|c|}{ Reason for missing school (N=121) } \\
\hline Pain & 100 & 82.6 \\
\hline Heavy flow & 16 & 13.2 \\
\hline Felt dizzy & 5 & 4.1 \\
\hline
\end{tabular}

In other words, girls from a high socio-economic class tend to achieve menarche at a younger age. Most of the respondents informed their mothers first at the achievement of menarche and it was their mothers also that educated majority of them on menarche and menstruation. This is in keeping with the family life as girls are known to have close relationship with their mothers especially on feminine matters. Such were also the findings in other studies. $5,7,8$

The modal menstrual flow duration in this study of 4 days is within the normal physiology. Only $0.6 \%$ of the students had abnormal flow duration of more than 7 days. In fact, $99.0 \%$ of them had normal duration of flow. However, $24.4 \%$ of the students missed school attendance on account of menstruation as was the case in other studies in India and Ghana and majority of them did so because of dysmenorrhoea. ${ }^{16,17}$ This will likely affect their academic performance in the long run.

It is surprising that there is no significant association between the duration of flow and development of tiredness (which suggests anaemia) as one would expect. However, long flow duration may not necessarily be accompanied by heavy loss which is what will lead to anaemia. Flow duration is not also related to the respondents' BMI, presence of dysmenorrhoea or cycle regularity.

\section{CONCLUSION}

The average age of menarche of secondary school girls in Awka was $12.77 \pm 1.159$ years. Most of the students had normal menstrual flow duration of 2-7 days with a cycle 
length of 21-35 days. Only a small minority had a heavy flow.

\section{ACKNOWLEDGMENTS}

Authors would like to thank the students who were the research assistants that helped in administering the questionnaires.

Funding: No funding sources

Conflict of interest: None declared

Ethical approval: The study was approved by the Institutional Ethics Committee

\section{REFERENCES}

1. Dharampal GD, Sanjay VW, Jayesh YD. Age at menarche and menstrual cycle pattern among school adolescent girls in central India. Glob J Health Sci; 2012;4(1):105-11.

2. Desalegen TZ, Berihun M, Abay M. Age at menarche and menstrual pattern of secondary school adolescents in northwest Ethiopia. BMC Women's Health. 2009;9:29.

3. Dare FO, Ogunniyi SO, Makinde OO. Biosocial factors affecting Menarche in a mixed Nigerian Population. Central African J Med. 1992;38(2):7781.

4. Dalton ME, Dalton K. Menarchal age in the disable. BMJ. 1978;2:475.

5. Ikaraoha C, Mbadiwe IC, Igwe CU, Allagua DO, Mezie O, Iwo GTO, et al. Menarcheal age of secondary school girls in urban and rural areas of rivers state, Nigeria. J Health Allied Sci. 2005;2:4

6. Garba I, Rabiu A, Abubakar I. Memstrual hygiene among adolescent school girls in Kano. Trop J Obstet Gynaecol. 2018;35(2):153-7.

7. Anikwe CC, Mamah JE, Okorochukwu BC, Nnadozie UU, Obarezi CH, Ekwedigwe KC. Age at menarche, menstrual characteristics, and its associated morbidities among secondary school students in Abakaliki, southeast Nigeria. Heliyon. 2020;6(5):45-9.

8. Diorgu FC, Diorgu KN. Average pubertal timing, characteristics and menstrual patterns of Nigerian girls: Implication for sex education . J Gynecol Res Obstet. 2020;6(1):10-3.

9. Padez C. Age at menarche of schoolgirls in Maputo, Mozambique. Ann Human Biol. 2003;30:487-95.

10. Montero P, Bernis C, Loukid M, Hilali K, Baali A. Characteristics of menstrual cycles in Moroccan girls: prevalence of dysfunctions and associated behaviours. Ann Human Biol. 1999; 26:249-3.

11. Shah V. A rite of passage: a mixed methodology study about knowledge, perceptions and practices of menstrual hygiene management in rural Gambia. BMC Public Health. 2019;19:277.

12. Lawan UM, Yusuf NW, Musa AB. Menstruation and menstrual hygiene amongst adolescent school girls in Kano, Northwestern Nigeria. Afr J Reprod Health. 2010;14:201-7.

13. Rabiu A, Abubakar IS, Garba I. Dysmenorrhea and menstrual patterns among adolescent school girls in Kano. N Niger J Clin Res. 2019;8:30-4.

14. Oyedeji GA. Socioeconomic and cultural background of hospitalized children in Ilesa. Niger $\mathbf{J}$ Paediatr. 1985;12:111-7.

15. Pandey M, Pradhan A. Age of attainment of menarche and factors affecting it amongst school girls of Gangtok, Sikkim, India. Ann Human Biol. 2017;4(6):2187-92.

16. Vashisht A, Pathak R, Agarwalla R, Patavegar B, Panda M. School absenteeism during menstruation amongst girls in Delhi, India. J Family Community Med. 2018;25(3):163-8.

17. Mohammed S, Larsen-Reindorf E, Awal I. Menstrual hygiene management and school absenteeism among adolescents in Ghana: results from a schoolbasedcross-sectional study in a rural community. Int J Reproduc Med. 2020;2020:1-9.

Cite this article as: Ezenyeaku CC, Ezebialu IU, Umeobika JC, Akabike J, Ezenyeaku CA, Ojiyi CE, et al. Assessment of age at menarche and menstrual pattern among secondary school girls in Awka, Southeast Nigeria. Int J Reprod Contracept Obstet Gynecol 2021;10:2192-7. 